\title{
Role of polymeric surfactants on the growth of manganese ferrite nanoparticles
}

\author{
Tahereh Rohani Bastami ${ }^{\text {a,b }}$, Mohammad H. Entezari ${ }^{b, *}$, Qiu Hong Hu ${ }^{a}$, Sandy Budi Hartono ${ }^{\text {a }}$ \\ Shi Zhang Qiao ${ }^{\mathrm{a}, *}$ \\ ${ }^{a}$ ARC Centre of Excellence for Functional Nanomaterials, Australian Institute for Bioengineering and Nanotechnology, The University of Queensland, QLD 4072, Australia \\ ${ }^{\mathrm{b}}$ Department of Chemistry, Ferdowsi University of Mashhad, 91775 Mashhad, Iran
}

\section{H I G H L I G H T S}

- The effect of polymeric surfactants was studied on the growth of manganese ferrite nanoparticles.

- Nanoparticles were formed by attachment and growth of primary building blocks.

- Particle size was dependent to the kind of surfactant and the time and temperature of the reaction.

- Nanoparticles were collapsed after reaching a critical size.

- More collapses between primary building blocks were observed by using PEG $_{10000}$.

\section{A R T I C L E I N F O}

\section{Article history:}

Received 13 May 2012

Received in revised form 2 August 2012

Accepted 9 August 2012

Available online 29 August 2012

\section{Keywords:}

Manganese ferrite

Growth kinetics

Polyethylene glycol

Ostwald ripening

\begin{abstract}
A B S T R A C T
The growth kinetics of manganese ferrite $\left(\mathrm{MnFe}_{2} \mathrm{O}_{4}\right)$ nanoparticles was studied by solvothermal reaction of iron and manganese salts in ethylene glycol as a solvent. To explore the mechanism of the nanoparticle formation and development, polyethylene glycol (PEG) with different molecular weights and polyvinyl pyrrolidone (PVP) were used as polymeric surfactants to investigate their effects on the formation of $\mathrm{MnFe}_{2} \mathrm{O}_{4}$ nanoparticles. The size evolution and the size distribution not only dependent on the kind of surfactant but also on the time and temperature of reaction process. In the presence of low molecular weight PEG ( $\mathrm{PEG}_{300}$ ), nanoparticles with diameter of $180 \mathrm{~nm}$ and narrow size distribution could be produced at $160{ }^{\circ} \mathrm{C}$ during $12 \mathrm{~h}$ of reaction while the nanoparticles with average size of $330 \mathrm{~nm}$ were formed by using $\mathrm{PEG}_{300}$ at $200{ }^{\circ} \mathrm{C}$ and $48 \mathrm{~h}$. Therefore, by increasing the temperature and the time of reaction, the size of nanoparticles was increased and finally reached a critical size and then collapsed. When a large molecular weight surfactant $\mathrm{PEG}_{1000}$ was used, the nanoparticles with average size of $230 \mathrm{~nm}$ were formed at $180^{\circ} \mathrm{C}$ and $60 \mathrm{~h}$. In the case of $\mathrm{PEG}_{300}$ and $\mathrm{PEG}_{10000}$ as lower and higher molecular weights, the separation between building blocks occurred after $60 \mathrm{~h}$ and $48 \mathrm{~h}$ for $180^{\circ} \mathrm{C}$ and $200{ }^{\circ} \mathrm{C}$, respectively. However, more collapses between primary building blocks were observed by using PEG $_{10000}$. The nanoparticles were composed of small building blocks and exhibited a spherical mesocrystal structure which was demonstrated from the TEM and scanning electron microscope (SEM) results. The investigation on the growth mechanism of the nanoparticles indicated that the formation of manganese ferrite was followed by the attachment and growth of primary building blocks and their Ostwald ripening process.
\end{abstract}

() 2012 Elsevier B.V. All rights reserved.

\section{Introduction}

Magnetic nanoparticles have attracted great interests [1-6]. One of the most important groups of magnetic nanoparticles is ferrite which has a very high magnetization value at room temperature [7]. Manganese ferrite $\left(\mathrm{MnFe}_{2} \mathrm{O}_{4}\right)$ as a superparamagnetic nanoparticle has a very high magnetization capacity owing to its

* Corresponding authors. Tel.: +98 5118797928 304; fax: +98 5118795457 (M.H. Entezari), tel.: +61 7 33463815; fax: +61 722463973 (S.Z. Qiao).

E-mail addresses: moh_entezari@yahoo.com (M.H. Entezari), s.qiao@uq.edu.au (S.Z. Qiao). large magnetic spin magnitude [8]. It has been widely used in electronic $[9,10]$, contrast-enhancement agents in MRI technology [1113] and recording media [14]. However, magnetic properties of the nanoparticles and its application are strongly dependent on the size, shape, morphology and crystallinity of the nanoparticles [15-19]. Manganese ferrite nanoparticles with different sizes and morphologies have been synthesized by different methods. Some of these methods include ball milling [20], co-precipitation of $\mathrm{Mn}^{2+}$ and $\mathrm{Fe}^{3+}$ in aqueous solution [21], reverse micelle [22,23], thermal decomposition [24-27], and solvothermal method [19]. The last method offers many advantages over the others such as its simplicity, high crystallinity of the products, capability to 
control the crystal growth and its adequacy for the preparation of large quantities of samples [19].

Most of the works focus on the synthesis of ferrite nanoparticles with sizes below $30 \mathrm{~nm}$ which is difficult to separate from solution or control their movement in blood by using moderate magnetic fields [28]. This limits their practical applications such as bioseparation and targeted drug delivery. To overcome this problem, Li and co-workers reported the synthesis of monodisperse $\mathrm{MFe}_{2} \mathrm{O}_{4}$ ferrite $(\mathrm{M}=\mathrm{Fe}, \mathrm{Co}, \mathrm{Mn}, \mathrm{Ni}, \mathrm{Zn}, \mathrm{Cu})$ microspheres by a solvothermal reaction through their partial reduction of the reagent by ethylene glycol (EG) in the presence of NaAc and polyethylene glycol (PEG) [29]. The produced particles are composed of smaller building blocks with superparamagnetic properties [30]. To control the morphology, shape and size, it is necessary to study the kinetics of nucleation and growth, and structure development of nanocrystals $[31,32]$.

It has been reported that the growth of particles undergoes two major stages, i.e., aggregation and coarsening [33]. This process typically starts with nuclei formation followed by its growth. In a heterogeneous solution, the coarsening process is driven by a decrease in surface energy. This process referred to as Ostwald ripening in which the growth of larger particles occurs at the expense of less stable smaller particles. Aggregation is achieved by the combination of primary particles into large secondary particles. In the oriented aggregation, primary crystallites combine to each other to form secondary crystals which are new single crystals composed of oriented subunits [33-36].

Recently, Niederberger and Coelfen [36] defined a "mesocrystal" as a particle composed of primary units in crystallographic registry but without coherent, crystalline material linking them [37]. In such an object, solvent molecules and/or other species are located in the spaces between the aligned crystallites.

The preparation of mesoscale nanoparticles occurs in several steps. Initially, primary nanoparticles (building blocks) are formed via a classical nucleation and crystal growth. Then, building blocks undergo oriented self-assembly process $[38,39]$. Three types of mesocrystal have been proposed [38]; M-I, the building blocks are isolated and only "bridged" via organic substances, that is, the original mesocrystal defined by Colfen; M-II, the building blocks are partly bridged by organic substances and partly by themselves; M-III, the building blocks are only bridged by themselves.

Surface properties of primary nanoparticles are crucial for determination of final structure of secondary particles. It is suggested that organic ligands with high binding affinities to primary nanoparticles can promote self-assembly by the adsorption of ligand instead of themselves, type (I) [40]. In the absence of sufficiently strong-surface protecting layers, it seems the primary nanoparticles would always randomly aggregate into disordered solids.

In this work, we present the effect of organic additives like polymeric surfactants on the growth process, size and morphology of manganese ferrite nanoparticles. The monodisperse manganese ferrites were synthesized in the presence of polymeric surfactants such as PEG with different molecular weights $\left(\mathrm{PEG}_{300}, \mathrm{PEG}_{6000}\right.$, and $\left.\mathrm{PEG}_{10000}\right)$ and polyvinyl pyrrolidone $\left(\mathrm{PVP}_{40000}\right)$ with different chemical structure. Our results show that the polymeric surfactants have crucial effects on the control of structure and morphology of the mesocrystal manganese ferrite nanoparticles. Based on our knowledge, this is the first report about the effect of organic additives on the growth process of magnetic nanoparticles.

\section{Materials and methods}

\subsection{Material}

Iron(III) chloride hexahydrate, manganese (II) acetate tetrahydrate, ethylene glycol anhydrous, sodium acetate anhydrous, and
PEG ( $\mathrm{PEG}_{300}, \mathrm{PEG}_{6000}$, and $\mathrm{PEG}_{10000}$ ) were purchased from SigmaAldrich. $\mathrm{MnCl}_{2} \cdot 4 \mathrm{H}_{2} \mathrm{O}$, Iron (III) acetylacetonate, and PVP were purchased from Fluka. Mili-Q water was used with a resistivity not less than $18.2 \mathrm{M} \Omega \mathrm{cm}^{-1}$.

\subsection{Preparation of $\mathrm{MnFe}_{2} \mathrm{O}_{4}$}

In the first experiment, the $\mathrm{MnFe}_{2} \mathrm{O}_{4}$ nanoparticles were prepared from modified $\mathrm{Li}$ et al. method [29]. Briefly, $0.25 \mathrm{~g}$ ( $2.5 \mathrm{mmol}$ ) of $\mathrm{MnCl}_{2} \cdot 4 \mathrm{H}_{2} \mathrm{O}$ and $0.67 \mathrm{~g}(5 \mathrm{mmol})$ of $\mathrm{FeCl}_{3} \cdot 6 \mathrm{H}_{2} \mathrm{O}$ were dissolved in ethylene glycol $(20 \mathrm{~mL})$ to obtain a yellow clear solution. Then $1.8 \mathrm{~g}$ of sodium acetate and $0.5 \mathrm{~g}$ of different polymeric surfactant $\left(\mathrm{PEG}_{6000}, \mathrm{PEG}_{10000}, \mathrm{PVP}\right.$ ) were added under vigorous stirring for $30 \mathrm{~min}$ at $80^{\circ} \mathrm{C}$ to melt the surfactant. In the case of $\mathrm{PEG}_{300}$, $0.44 \mathrm{~mL}(0.5 \mathrm{~g})$ of surfactant was added under vigorous stirring for $30 \mathrm{~min}$ at room temperature. After that, the prepared solution was sealed in a Teflon-lined stainless-steel autoclave and maintained at three different temperatures $\left(160^{\circ} \mathrm{C}, 180^{\circ} \mathrm{C}, 200^{\circ} \mathrm{C}\right)$ and different time intervals, and then allowed to cool to room temperature. The products were washed three times with ethanol and dried at $50{ }^{\circ} \mathrm{C}$ for overnight.

The reactants with organic anions were also used to prepare the $\mathrm{MnFe}_{2} \mathrm{O}_{4}$ nanoparticles. Briefly, $0.306 \mathrm{~g}$ ( $\left.2.5 \mathrm{mmol}\right)$ of manganese

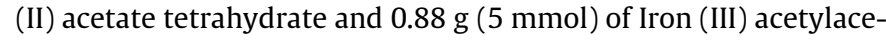
tonate were dissolved in ethylene glycol $(20 \mathrm{~mL})$ to obtain a clear solution. Then $1.8 \mathrm{~g}$ of sodium acetate and $0.5 \mathrm{~g}$ of $\mathrm{PEG}_{6000}$ were added under vigorous stirring for $30 \mathrm{~min}$ at $80^{\circ} \mathrm{C}$ to melt the surfactant. After that, the prepared solution was sealed in a Teflonlined stainless-steel autoclave and maintained at $200{ }^{\circ} \mathrm{C}$ in different time intervals (4-20 h), and then allowed to cool to room temperature. The products were washed three times with ethanol and dried at $50{ }^{\circ} \mathrm{C}$ for overnight.

\subsection{Characterization}

The size and morphology of the obtained $\mathrm{MnFe}_{2} \mathrm{O}_{4}$ nanoparticles were characterized using a JEOL-1010 TEM operating at $100 \mathrm{kV}$ of accelerate voltage. The TEM sample was prepared by dispersing nanoparticles in ethanol via ultrasound and dropped on the copper grid before loading to the instrument. The SEM is carried out by JSM 6300 (JEOL) with $5 \mathrm{kV}$ of accelerate voltage. The specimen was treated with platinum coating prior to loading into SEM instrument. The X-ray diffraction (XRD) was used to identify the crystal structure and the average size of the nanoparticles. The XRD was performed using Rigaku-Miniflex equipped with Co radiation $(\lambda=1.7902 \AA)$ in the wide range of Bragg's angle $\left(15-80^{\circ}\right)$. The average crystallite size was calculated by Debye-Sherrer's equation [41]. To understand the adsorption mechanism of organic additives (polymeric surfactants), the Fourier infrared (FTIR) measurements were carried out on the pure surfactants and the synthesized $\mathrm{MnFe}_{2} \mathrm{O}_{4}$ nanoparticles in the presence of surfactants. The FTIR of the samples were recorded using Nicolet 6700 in the range of $400-4000 \mathrm{~cm}^{-1}$ with ATR accessories. The thermogravimetric analysis (TGA) was performed for samples ( $30 \mathrm{mg}$ ) with a heating rate of $5^{\circ} \mathrm{C} / \mathrm{min}$ using a Mettler Toledo TGA thermogravimetric analyzer in $\mathrm{N}_{2}$ atmosphere up to $800^{\circ} \mathrm{C}$.

\section{Results and discussion}

Manganese ferrites were obtained via co-precipitation of $\mathrm{Mn}(\mathrm{II})$ and $\mathrm{Fe}(\mathrm{III})$ ions in solvothermal condition. The iron(III) and manganese (II) with organic and inorganic anions were acted as precursors, sodium acetate and alkaline agents as electrostatic stabilizer, and ethylene glycol as high boiling point solvent $\left(196-198{ }^{\circ} \mathrm{C}\right)$ and reducing agent [42]. Different polymeric surfactants were used as 
organic additives for assembling the smaller building blocks to produce nanosphere. The nearly mono-dispersed nanoparticles were obtained by using manganese (II) and iron (III) chloride salts.

\subsection{Particle size and distribution}

\subsubsection{Precursor with organic anion}

The sizes of nanoparticles prepared with precursors of iron and manganese compounds containing organic anions were 50 and $80 \mathrm{~nm}$ at aging times of 4 and $8 \mathrm{~h}$, respectively. By increasing the time to $10 \mathrm{~h}$, the structure was collapsed and the building blocks were separated (Fig. S1). As it is shown in Fig. S1, the structure and morphology of the particles formed by organic anion were more disorder than particles formed by chloride as precursor's anion. The average size of magnetic nanoparticles was 185, 200 and $220 \mathrm{~nm}$ after 4,8 , and 12 h by chloride as anion. The main hypothesis is that the organic anions play a role the same as co-surfactant and prohibit the effective contact between particles and oriented attachment at the interface of primary particles in the present of polymeric surfactants. Furthermore, the growth of building blocks via Ostwald ripening is prohibited. Therefore, the final size of nanoparticles was smaller than the particles produced by mineral anion and the structure was more disorder.

Two crucial points for these observations were proposed: (1) bridging between building blocks occurs via polymeric surfactant and organic anions which adsorbed on the surface of nanoparticles. Both of sorbates should be unstable under hydrothermal conditions. As desorption of adsorbed layer increases, the separation between primary building blocks increases too. Therefore, bridging between primary building blocks should be more unstable when using organic anions as precursors. For this reason, in the case of organic precursors the structure of nanoparticles was collapsed after $10 \mathrm{~h}$. (2) the higher movement of primary nanoparticles can be obtained under thermal conditions due to irreversible desorption of binder [31].

\subsubsection{Precursor with inorganic anion}

The particle size, size distribution, and nanoparticle structure were determined by TEM analyses. For the manganese ferrites prepared from chloride anions the following points can be discussed:

1. Fig. 1 shows the relationship between the average particle size and the aging time under different temperatures $(160,180$, and $200{ }^{\circ} \mathrm{C}$ ) in the presence of $\mathrm{PEG}_{300}$ or $\mathrm{PEG}_{10000}$. Fig. 2 presents the TEM images of manganese ferrite prepared in the presence of $\mathrm{PEG}_{300}$ at different temperatures and constant aging time $(12 \mathrm{~h})$. The structure and size distribution were more uniform at lower temperatures and shorter aging times and the average size was $180 \mathrm{~nm}$ at $160^{\circ} \mathrm{C}$.

2. At low temperature $\left(160^{\circ} \mathrm{C}\right)$ the particle size increases with increasing the time. But, at higher temperatures, 180 and $200^{\circ} \mathrm{C}$, a collapse was observed in longer aging times. The time for the collapse was shorter for the samples prepared at $200{ }^{\circ} \mathrm{C}$ than $180^{\circ} \mathrm{C}$. These observations could be explained by the partial removal of polymeric surfactants at higher temperatures and longer aging times. Under these conditions the interaction forces between building blocks are low and their kinetic energies are high, and therefore a collapse can be occurred easily.

3. At the same temperature, the average particle size of the magnetic nanoparticles obtained by $\mathrm{PEG}_{300}$ is higher than by $\mathrm{PEG}_{10000}$. This is might be related to the lower molecular weight of PEG with lower tendency to the building blocks than higher molecular weight of PEG. Therefore, larger particles can be obtained in the presence of $\mathrm{PEG}_{300}$. When organic molecules show strong and high binding affinity to the surface of nanoparticles, the nanoparticles are highly stabilized and further crystal growth becomes harder. These organic molecules impose a ste-

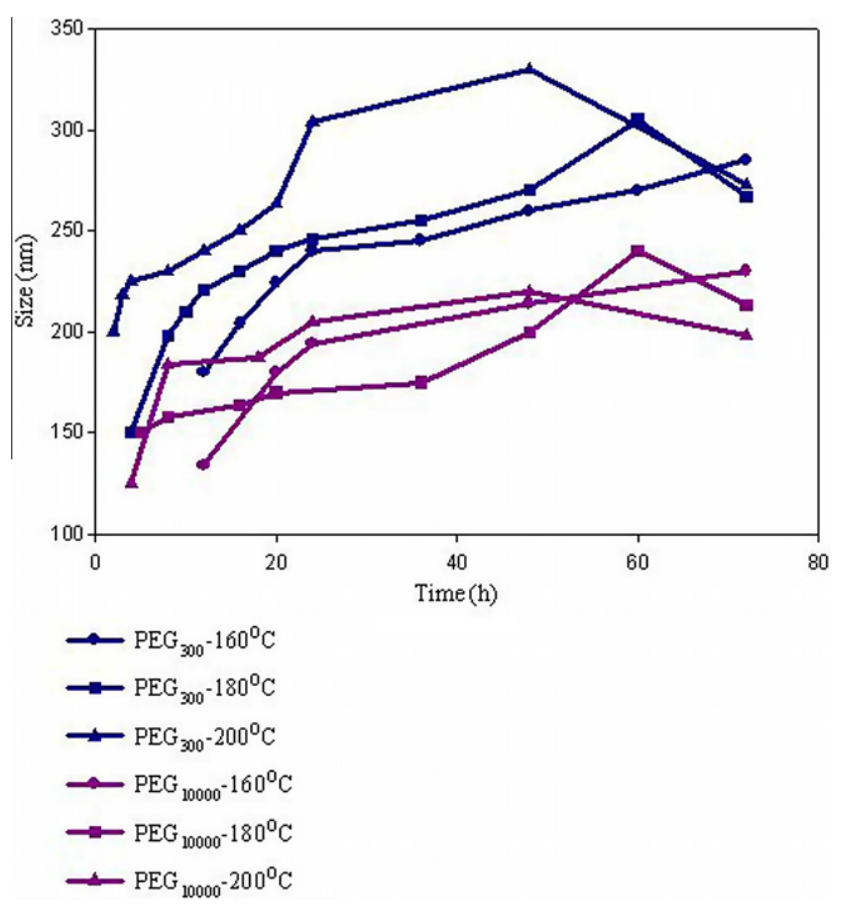

Fig. 1. TEM data for the size versus time at different temperatures.

ric effect at the interface of primary nanoparticles which hinder the oriented attachments by preventing the effective particleparticle contact and reduction of the Brownian motion. Also, the presence of organic molecule at the surface of building blocks can prevent the growth by Ostwald ripening process.

4. The nanoparticles were broken by increasing the temperature. Fig. S2 illustrates the separation between primary building blocks at $200{ }^{\circ} \mathrm{C}$ in $72 \mathrm{~h}$ for $\mathrm{PEG}_{6000}$ and PVP. In the case of $\mathrm{PVP}$, some of the nanoparticles were broken after $24 \mathrm{~h}$ at $200{ }^{\circ} \mathrm{C}$ and the TEM does not show a complete collapse like $\mathrm{PEG}_{6000}$.

5. Some hollow sphere structures can be seen at $180^{\circ} \mathrm{C}$ and $200{ }^{\circ} \mathrm{C}$ when PEG was used as a surfactant (Fig. S3). However, in the case of PVP, Fig. 3 shows the nanoparticles synthesized at $160^{\circ} \mathrm{C}, 180^{\circ} \mathrm{C}, 200^{\circ} \mathrm{C}$ and clearly presented the hollow sphere structure of magnetic nanoparticle in Fig. 3c. It is assumed that the small vacancies trapped inside the particle can migrate and formed a larger vacancy inside the particle. This situation can occur faster at higher temperatures which the building blocks have higher movements. Under these conditions, the small vacancies with high surface energy changes to a large vacancy with low surface energy. When the aging time is short and the temperature is low the manganese ferrite has a solid core (entirely dark) [43]. The TEM images confirm that the hollow structure increased with increasing of the temperature (Fig. S4).

6 . The temperature and the type of surfactant play a crucial role in the induction time of the nanosphere formation. The induction times at $160{ }^{\circ} \mathrm{C}, 180^{\circ} \mathrm{C}$, and $200^{\circ} \mathrm{C}$ for $\mathrm{PEG}_{300}$ are about 12,5 , and $2 \mathrm{~h}$, respectively. Induction time is longer for the lower temperature. The induction time is also changed with different types of surfactants as illustrated in Table 1. It is confirmed that the lower induction time can be observed for the manganese ferrite obtained by using $\mathrm{PEG}_{300}$.

\subsection{Kinetic growth study}

The entire XRD diffraction peaks match well with the pattern of cubic structure of $\mathrm{MnFe}_{2} \mathrm{O}_{4}$ (ICDD no.10-0319). According to the 

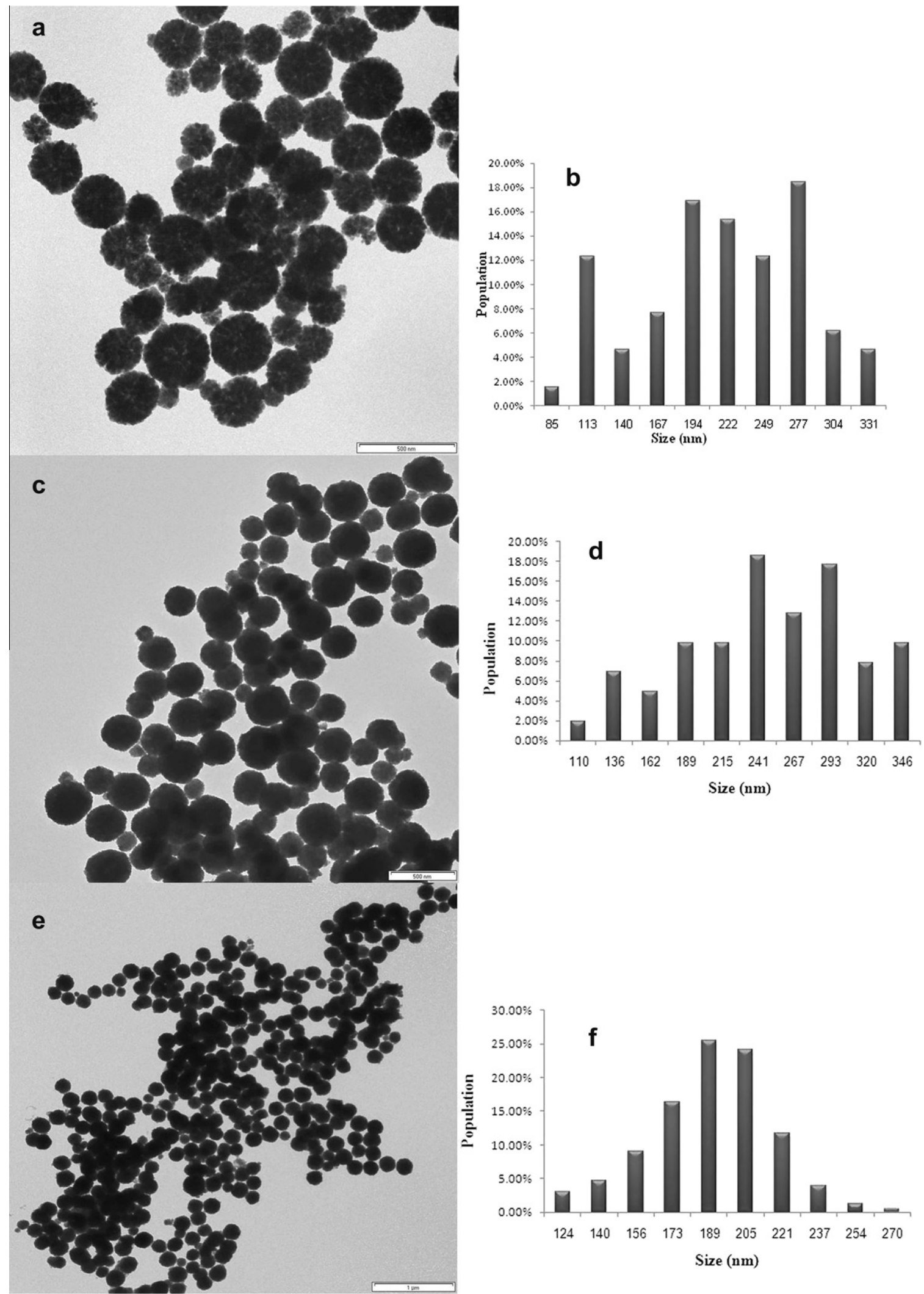

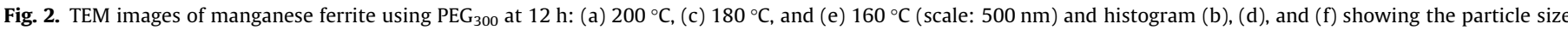
distribution measured from (a), (c), and (e), respectively.

literature $[28,30]$, the grain size of the nanoparticles can be obtained based on the Debye-Scherrer formula. The XRD results show that the size of building blocks of the nanoparticles increases with increasing the time and temperature. Fig. 4 presents the XRD patterns of the manganese ferrite nanoparticles prepared by $\mathrm{PEG}_{300}$ at $200^{\circ} \mathrm{C}$ under different aging times. The crystallinity 

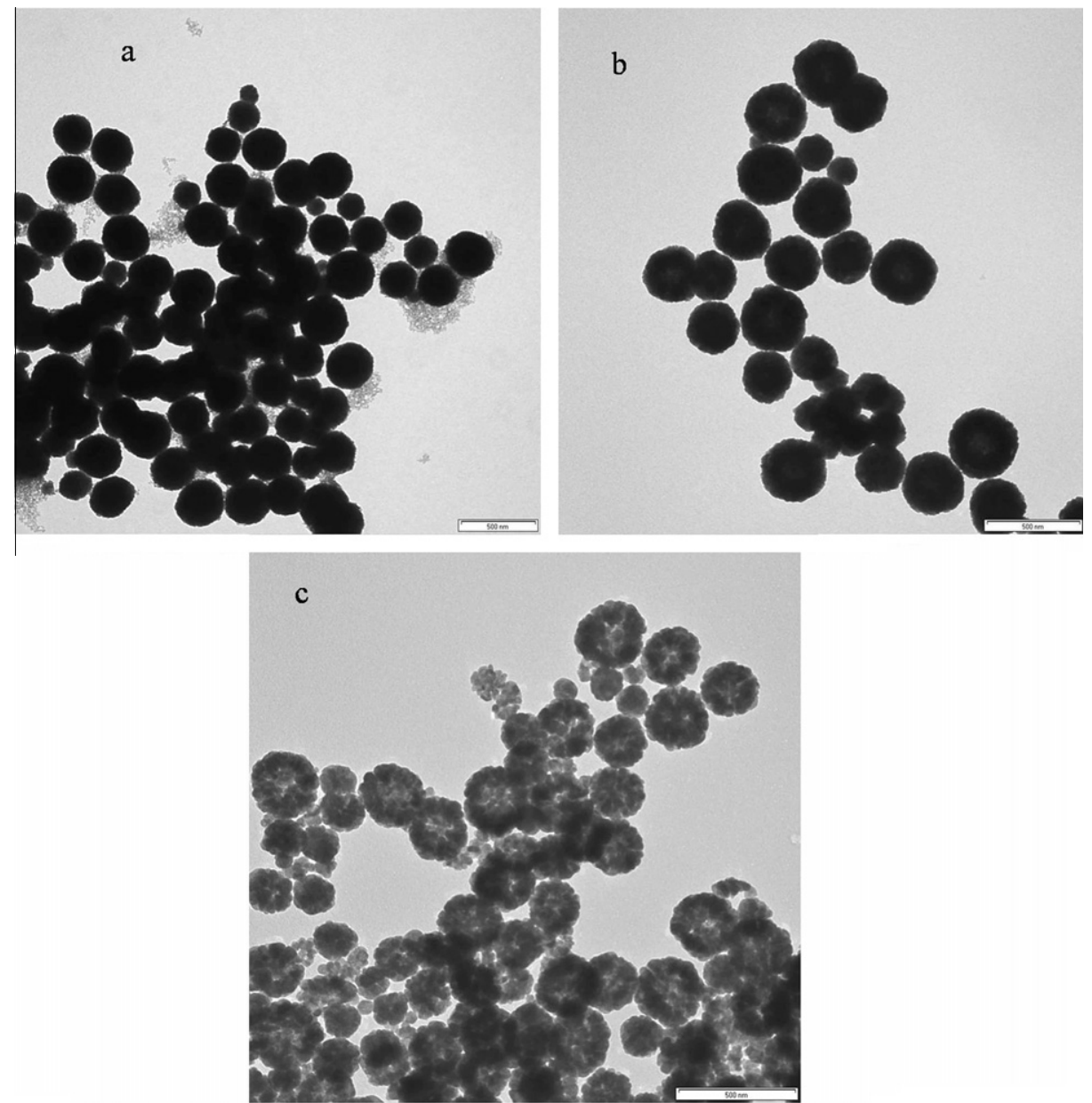

Fig. 3. TEM images of nanoparticle with PVP as a surfactant: (a) $160^{\circ} \mathrm{C}, 20 \mathrm{~h}$; (b) $180^{\circ} \mathrm{C}, 16 \mathrm{~h}$; and (c) $200{ }^{\circ} \mathrm{C}, 16 \mathrm{~h}(\mathrm{scale}$ : $500 \mathrm{~nm}$ ).

Table 1

Estimated values by fitting the experimental data at each temperature.

\begin{tabular}{|c|c|c|c|c|c|c|c|c|c|c|c|c|}
\hline \multirow{2}{*}{$\frac{\text { Surfactant }}{t\left({ }^{\circ} \mathrm{C}\right)}$} & \multicolumn{3}{|c|}{$\mathrm{PEG}_{300}$} & \multicolumn{3}{|c|}{$\mathrm{PEG}_{6000}$} & \multicolumn{3}{|c|}{$\mathrm{PEG}_{10000}$} & \multicolumn{3}{|c|}{$\mathrm{PVP}_{40000}$} \\
\hline & 160 & 180 & 200 & 160 & 180 & 200 & 160 & 180 & 200 & 160 & 180 & 200 \\
\hline$D_{0}(\mathrm{~nm})$ & 5 & 6 & 9 & - & 7 & 13 & 7 & 5.5 & 7 & 11 & 7 & 10 \\
\hline$t_{0}(\mathrm{~h})$ & 10 & 4 & 2 & 12 & 8 & 4 & 12 & 5 & 3 & 12 & 5 & 3 \\
\hline$k_{c}\left(\mathrm{~h}^{-1 / 3}\right)$ & 4.88 & 5.25 & 6.52 & 3.71 & 4.75 & 5.50 & 2.75 & 4.35 & 4.92 & 3.58 & 4.43 & 4.72 \\
\hline$n$ & 2.91 & 2.60 & 3.10 & 2.52 & 2.87 & 3.32 & 2.75 & 2.60 & 2.52 & 2.82 & 2.62 & 2.97 \\
\hline$R_{\mathrm{sqr}}$ & 0.98 & 0.99 & 0.98 & 0.99 & 0.99 & 0.99 & 0.99 & 0.98 & 0.98 & 0.99 & 0.98 & 0.98 \\
\hline
\end{tabular}

was increased with the increase of aging time. At initial times $(2 \mathrm{~h})$, small building blocks are exist in the matrix (Fig. S5) and the XRD pattern shows less crystallinity and smaller size of primary building blocks. With increase of aging time, the small building blocks disappear and the intensity of peaks increases drastically. This confirms the increase of crystallinity of the nanoparticles after aggregation of primary building blocks.

It is obvious that the primary nanoparticles are produced by attachment of molecule/ion on single crystal and then the primary dispersed nanoparticles aggregate to each other by oriented attachment mechanism to obtain a secondary structure. The attachment of small building blocks on the surface of nanoparticles occurred in initial aging times (Fig. S5 and Table 1). In comparison with dispersed small nanoparticles, the aggregated structure is well oriented and crystallized. After that the growth of secondary nanoparticles followed by the Ostwald ripening mechanism. This is confirmed by the XRD results through the increase of nanoparti-

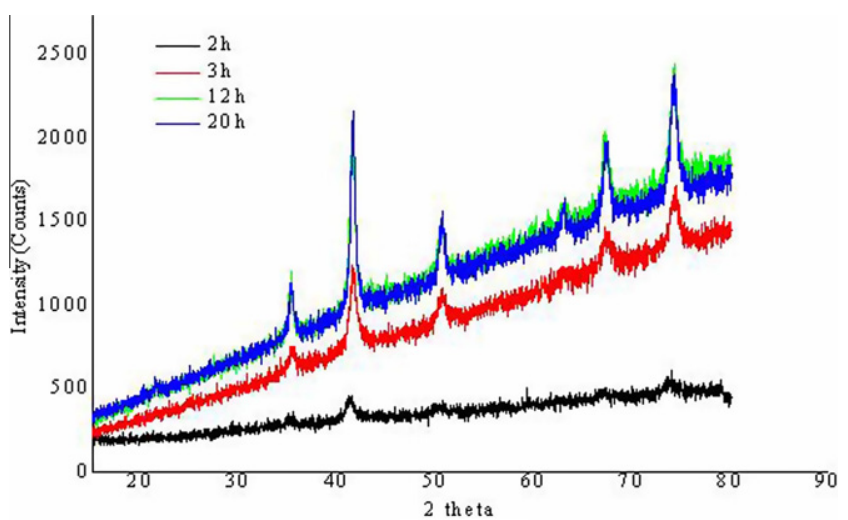

Fig. 4. XRD patterns of manganese ferrite using $\mathrm{PEG}_{300}\left(200^{\circ} \mathrm{C}\right)$. 
cle size and the SEM results for the growth of primary building blocks with increasing aging time (Fig. S6). This mechanism is in agreement with other research works $[28,40]$.

To study the kinetics of the growth process, it is necessary to determine the particle size of small nanopartilces as a function of time in different temperatures. Three kinetic models have been widely used to investigate the growth process of small building blocks: oriented attachment, simultaneous oriented attachment and coarsening or Ostwald ripening models which described by the three following equations, respectively:

$D_{t}=\frac{D_{0}\left(\sqrt[3]{2} k_{1} t+1\right)}{\left(k_{1} t+1\right)}$

$D_{t}=\frac{D_{0}\left(\sqrt[3]{2} k_{1} t+1\right)}{\left(k_{1} t+1\right)}+k_{2} t^{1 / n}$

$\left(D_{t}-D_{0}\right)=k_{c}\left(t-t_{0}\right)^{1 / n}$

The growth kinetics of all investigated samples was fitted well by the Ostwald ripening model (Eq. (3)). In this equation, $D_{t}$ and $D_{0}$ are the mean particle size at time $t$ and $t_{0}$, respectively and $k_{c}$ is a constant at certain temperature and $\mathrm{n}$ is an exponent relevant to the coarsening mechanism. For $n=2$, the coarsening mechanism is limited by precipitation/dissolution reaction at the particle matrix interface. For $n=3$, the coarsening kinetic is limited by the volume diffusion of ion in the matrix. When $n=4$, the coarsening kinetic is limited by the diffusion along the matrix boundary [33].

Table 1 shows the fitting results for the Eq. (3). As it is shown, the rate of growth was increased with decreasing the molecular weight of PEG. It is assumed that the binding of PEG to the surface of manganese ferrite is substantially stronger with higher molecular weight of PEG. To further investigate the growth mechanism, the activation energies $\left(E_{a}\right)$ for the growth process were determined. This is carried out by the temperature dependence of $k_{c}$ using the Arrhenius equation.

$\log k=-\frac{E_{a}}{R T}+A_{0}$

where $E_{a}$ is the apparent activation energy, $A_{0}$ is the pre-exponential factor, $R$ is the universal gas constant, and $T$ is the absolute temperature. The activation energy was found as $E_{a(\text { PEG300) }}=11.84 \mathrm{~kJ} / \mathrm{mol}$, $E_{a(\mathrm{PEG} 6000)}=17.07 \mathrm{~kJ} / \mathrm{mol}, \quad E_{a(\mathrm{PEG} 10000)}=26.91 \mathrm{~kJ} / \mathrm{mol}, \quad$ and $E_{a(-}$ $\mathrm{PVP} 40000)=12.46 \mathrm{~kJ} / \mathrm{mol}$.

One of the important parameters for the growth of nanoparticles is ripening growth of building blocks. It is assumed that the strong capping agents cause to hinder the Ostwald ripening growth. Therefore, the rate of Ostwald ripening is slower for PEG with higher molecular weight. In the coarsening equation, when $n=3$ the coarsening kinetics are limited by the volume diffusion of ion in the matrix. According to Table $1, n$ is about 3 and therefore, the ion or atom diffusion in solid matrix interface is closely related to the growth process and this step hinders by the adsorbed surface of nanoparticles using surfactant. It is revealed that the activation energy depends on the molecular weight and chemical structure of the surfactants. Desorption of PEG with higher molecular weight from the surface of nanoparticles needs more energy than $\mathrm{PEG}_{300}$. Therefore, it seems that $\mathrm{PEG}_{300}$ is more desorbed from the surface than the other surfactants.

\subsection{Surface characterization and crystal growth}

The FTIR and TGA analysis were carried out on different samples. These analyses can help to interpret the role of surfactant as organic binder on the surface of primary building blocks and their effects on the growth process.

\subsubsection{Analysis of FTIR}

Figs. 5, S7 and S8 show the FTIR spectrum of manganese ferrite obtained in the presence of $\mathrm{PEG}_{300}, \mathrm{PEG}_{10000}$, PVP as surfactants and some vibration results are summarizes in Table 2. According to the results, there are red shifts (increased wavelength) for the surfactants adsorbed on the nanoparticle surface due to their interactions with surface of nanoparticle. After adsorption, most of the peaks show blue shifts with increasing the time and temperature. This observation confirms the weakening of the adsorbed surfactants at higher thermal energy. More peaks disappeared with increasing the time and temperature which is related to the desorption of surfactants from the surface of nanoparticle at high thermal conditions. These observations confirm the mechanism of growth by desorption of surfactants from the surface of primary nanoparticles to obtain secondary structures. In addition, the collapse of nanoparticles at higher temperatures and longer times can be interpreted by these observations too.

The FTIR results also confirm the presence of adsorbed water molecule on the surface of nanoparticle at low temperature and short aging time. In the case of adsorbed PVP on the manganese ferrite, there are two peaks at $1570 \mathrm{~cm}^{-1}$ and a shoulder at $1670 \mathrm{~cm}^{-1}$. It is suggested that the interaction between surface of nanoparticle and PVP led to red shift of peak from $1670 \mathrm{~cm}^{-1}$ to $1570 \mathrm{~cm}^{-1}$ which is related to $\mathrm{C}=0$ stretching. A shoulder at $1670 \mathrm{~cm}^{-1}$ can be attributed to some uncoordinated surfactants on the surface of nanoparticle. At longer reaction time $\left(160^{\circ} \mathrm{C}\right.$ $72 \mathrm{~h}$ ), a blue shift to $1650 \mathrm{~cm}^{-1}$ was observed which is due to weakening of the adsorbed surfactant. Two other new peaks were appeared in the region $1050-1080 \mathrm{~cm}^{-1}$ and $879 \mathrm{~cm}^{-1}$ for PVP/ $\mathrm{MnFe}_{2} \mathrm{O}_{4}$. These peaks could be assigned for $\mathrm{C}-\mathrm{O}$ bond originated from EG.

The PEG molecules interact with $\mathrm{MnFe}_{2} \mathrm{O}_{4}$ surface via its $\mathrm{C}-\mathrm{O}-\mathrm{C}$ groups. The corresponding peak appears at $1100 \mathrm{~cm}^{-1}$

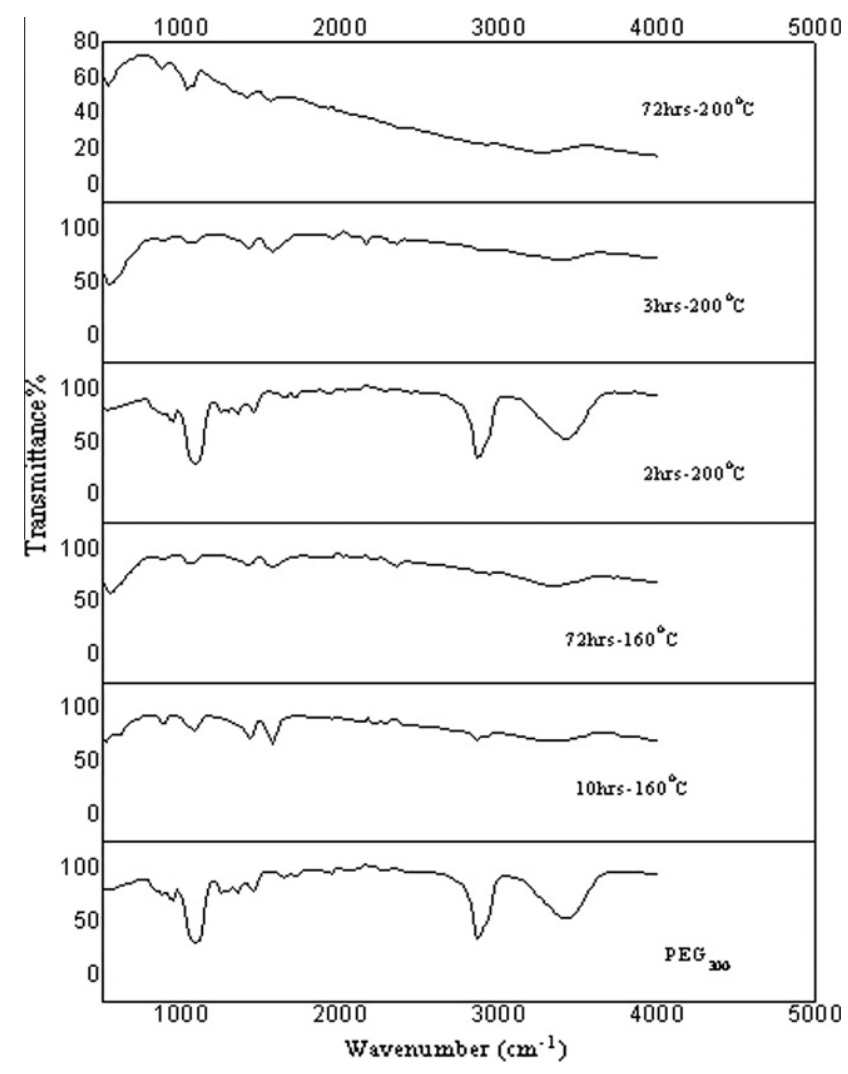

Fig. 5. FTIR spectra of $\mathrm{MnFe}_{2} \mathrm{O}_{4}$ using $\mathrm{PEG}_{300}$. 


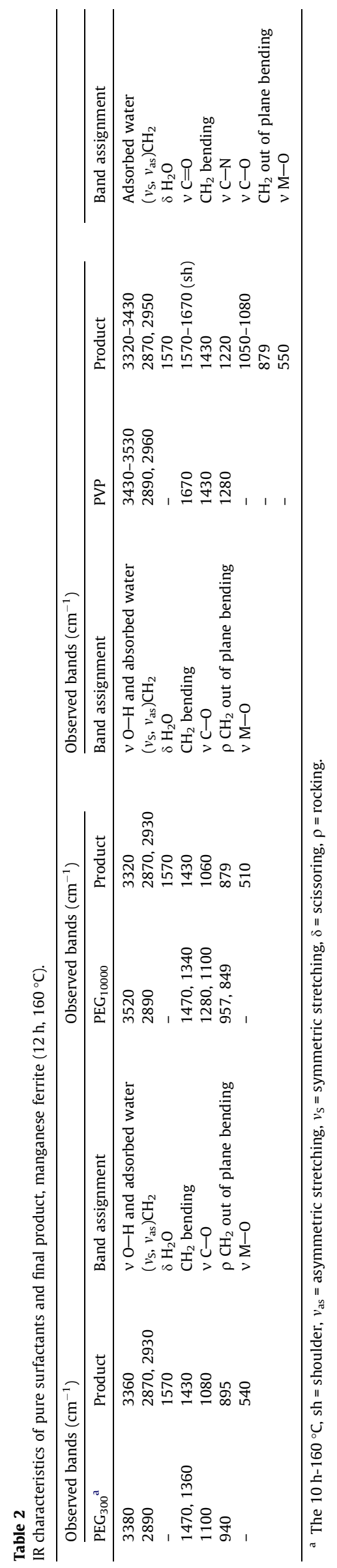

for pure PEG. Therefore, a shift can be seen from $1100 \mathrm{~cm}^{-1}$ to 1080 , and to $1060 \mathrm{~cm}^{-1}$ for $\mathrm{PEG}_{300}$ and $\mathrm{PEG}_{10000}$, respectively. This observation confirms the stronger binding between surface of manganese ferrite nanoparticle and $\mathrm{PEG}_{10000}$ than $\mathrm{PEG}_{300}$ which confirms the slower growth rate of primary nanoparticles in the presence of $\mathrm{PEG}_{10000}$. At $160{ }^{\circ} \mathrm{C}$ and $72 \mathrm{~h}$, this peak shifted to $1100 \mathrm{~cm}^{-1}$.

\subsubsection{Analysis of TGA}

The TGA curves provide addition evidences about the interaction of nanoparticles and surfactants. In this method the magnetic nanoparticles are heated to $800{ }^{\circ} \mathrm{C}$ under $\mathrm{N}_{2}$ and recorded the changes in mass due to the loss of organic material from the synthesized nanoparticles. Generally, the organic materials with strong bond to the particles can be desorbed at higher temperatures [44].

Figs. 6-8 and Table 3 show the TGA analysis of manganese ferrite nanoparticles under different preparation conditions. For all samples, the total loss of mass decreased with increasing the reaction time and temperature during the synthesis process. This observation is in agreement with the results obtained for the growth kinetic and FTIR analysis. The decomposition temperature of all pure surfactants is in the vicinity of $350{ }^{\circ} \mathrm{C}$. In all cases, the initial weight loss is attributed to the loss of surface-adsorbed water. In the case of $\mathrm{PEG}_{300}$, three weight losses were observed for the sample prepared at $10 \mathrm{~h}$ and $160{ }^{\circ} \mathrm{C}$. The first loss $(\sim 6 \%)$ is related to the loss of water from the surface of nanoparticles. This loss was occurred between the room temperature and $250^{\circ} \mathrm{C}$. The second loss $(\sim 14 \%)$ was observed in the range of $250-310^{\circ} \mathrm{C}$. The third loss was appeared between $540{ }^{\circ} \mathrm{C}$ and $800^{\circ} \mathrm{C}$. The TEM image (Fig. S5) shows a lot of primary building blocks nanoparticles in the sample at the initial time of reaction. This situation leads to loss a higher weight. After complete aggregation of the building blocks at higher temperatures and longer times, the amount of desorption significantly decreased. Some researchers explained that the TGA behaviors are based on the bi-layer adsorbed models on the particle surface $[45,46]$, while others claimed that the double-stepped TGA curve is due to two different kinds of binding sites $[47,48]$. Based on experimental results, it seems that the TGA curves for our samples are in agreement with the two different binding sites on the surface of the nanoparticles. As presented in all TGA curves, the double-stepped curve can be shown clearly in shorter times. Weight loss at the second section is much higher than the third one. This means that the concentration of adsorbing species with strong interaction is much lower than the concentration with lower interaction. In longer times, the third loss almost disappeared and the second loss was reduced. It should be mentioned that in longer times and higher temperatures, the chance of removal of adsorbed species with strong interaction (third loss) is lower than the species with lower interaction (second loss). But, the third loss is approximately disappeared in shorter time which is due to its low concentration.

According to Table 3 , the sample synthesized at $160^{\circ} \mathrm{C}$ for $12 \mathrm{~h}$ with $\mathrm{PEG}_{10000}$ acts similar to the sample obtained in $10 \mathrm{~h}$ and $160{ }^{\circ} \mathrm{C}$ with $\mathrm{PEG}_{300}$. An easier desorption of $\mathrm{PEG}_{300}$ can be assumed from the surface of nanoparticle than $\mathrm{PEG}_{10000}$. The total weight losses for $\mathrm{PEG}_{300}$ in $160^{\circ} \mathrm{C}$ and aging time of $10 \mathrm{~h}$ and $12 \mathrm{~h}$ are $26 \%$ and $16 \%$, respectively. For $\mathrm{PEG}_{10000}$ and PVP in $160{ }^{\circ} \mathrm{C}$ and $12 \mathrm{~h}$, the weight losses are about $41 \%$ and $50 \%$, respectively. The high weight losses at the initial time of reaction confirm the presence of large amount of surfactants on the surface of building blocks. In addition, the steric effect can be observed more for the cases of PVP and $\mathrm{PEG}_{10000}$. In the presence of all surfactants, the weight loss reduced significantly after complete aggregation of primary building blocks. TGA results indicated that 

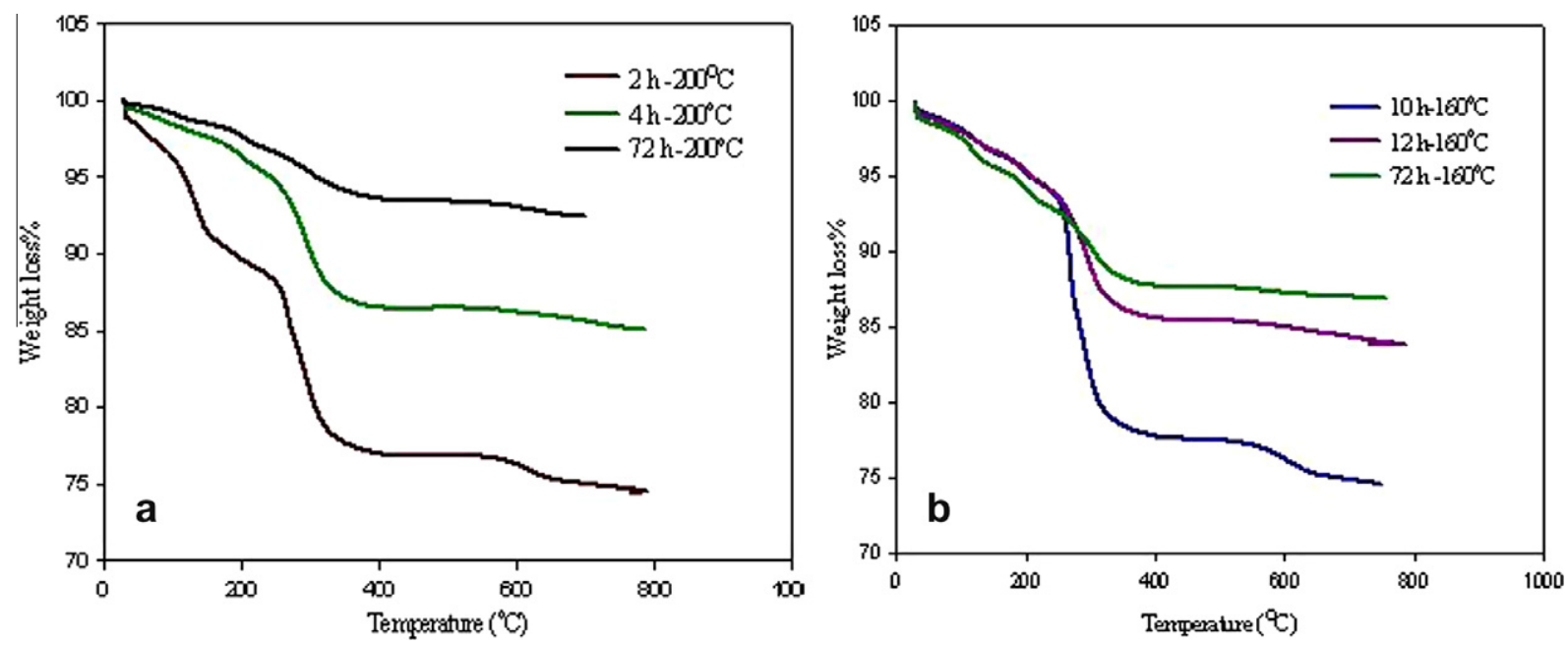

Fig. 6. The weight loss as a function of temperature (TGA) for $\mathrm{MnFe}_{2} \mathrm{O}_{4}$ nanoparticles with $\mathrm{PEG}_{300}$ for different time (a) $200{ }^{\circ} \mathrm{C}$, (b) $160{ }^{\circ} \mathrm{C}$.

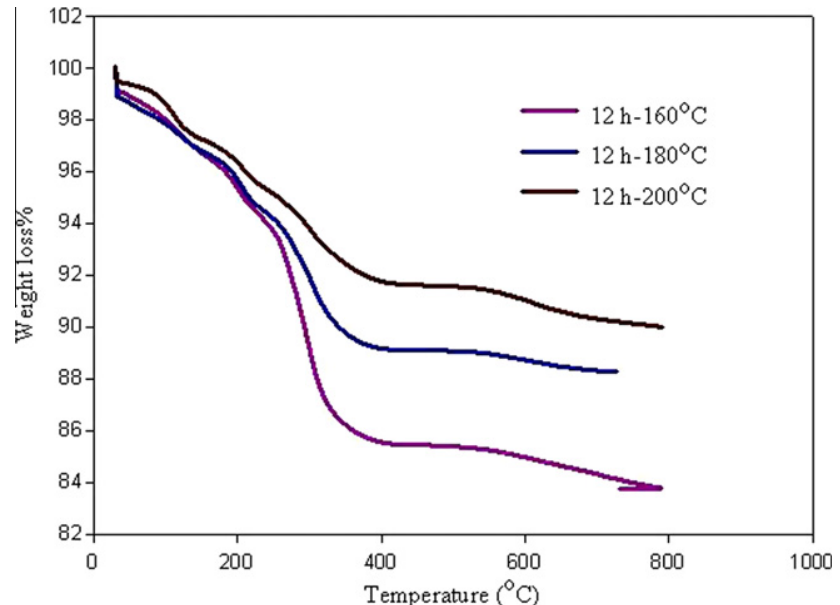

Fig. 7. TGA results for the weight loss as a function of temperature for $\mathrm{MnFe}_{2} \mathrm{O}_{4}$ nanoparticles with $\mathrm{PEG}_{300}$ in different temperature.

the adsorbed polymeric surfactants act as organic binder to assembly of primary building blocks. Also, less affinity of organic binder to the surface of primary building blocks led to their effective contact and production of aggregated nanoparticles in shorter aging time and temperature with narrower size distribution.

\section{Conclusion}

The growth of $\mathrm{MnFe}_{2} \mathrm{O}_{4}$ mesocrystal nanoparticles was studied by PVP and PEG with different molecular weights $\left(\mathrm{PEG}_{300}, \mathrm{PE6}_{000}\right.$, and $\mathrm{PEG}_{10000}$ ). Parameters such as induction time, stability of

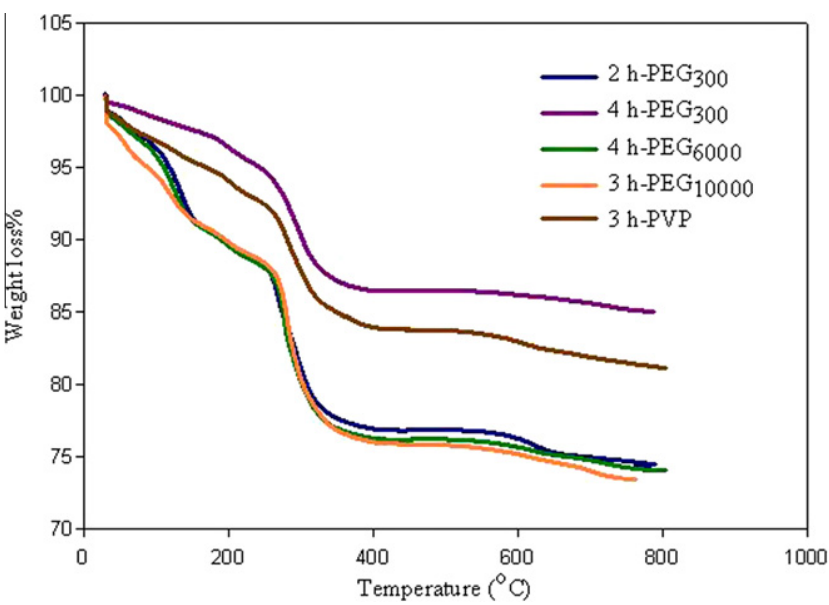

Fig. 8. The weight loss as a function of temperature (TGA) for $\mathrm{MnFe}_{2} \mathrm{O}_{4}$ nanoparticles with different surfactants $\left(200^{\circ} \mathrm{C}\right)$.

nanoparticles, size distribution, and uniformity of the particles were affected by different polymeric surfactants. At low temperature $\left(160^{\circ} \mathrm{C}\right)$ the particle size increased with increasing the aging time. However, a collapse was observed at higher temperatures $\left(180^{\circ} \mathrm{C}\right.$ and $\left.200^{\circ} \mathrm{C}\right)$. The time for the collapse was shorter at $200^{\circ} \mathrm{C}$ than $180^{\circ} \mathrm{C}$. Under some conditions the hollow sphere structures were observed clearly for the nanoparticles obtained with PVP. The TEM images confirm more hollow spheres with increasing the temperature. The final particle size was also increased by using the low molecular weight surfactant. The experimental data was fitted to the coarsening model and $n$ was about 3 which is consistent with the volume diffusion-limited coarsening.

Table 3

Results and analysis based on TGA curves for manganese ferrite nanoparticle.

\begin{tabular}{llll}
\hline Sample condition & Second weight loss (\%) & Third weight loss (\%) & Total mass loss + adsorbed water \\
\hline $12 \mathrm{~h}-160{ }^{\circ} \mathrm{C}-\mathrm{PVP}$ & 31 & 5 & 50 \\
$10 \mathrm{~h}-160^{\circ} \mathrm{C}-\mathrm{PEG}_{300}$ & 19 & 3 & 26 \\
$12 \mathrm{~h}-160^{\circ} \mathrm{C}-\mathrm{PEG}_{300}$ & 12 & 1.7 & 16 \\
$12 \mathrm{~h}-160^{\circ} \mathrm{C}-\mathrm{PEG}_{10000}$ & 28 & 4 & 41 \\
$72 \mathrm{~h}-160^{\circ} \mathrm{C}-\mathrm{PVP}$ & 6 & 2 & 11 \\
$72 \mathrm{~h}-160^{\circ} \mathrm{C}-\mathrm{PEG}_{300}$ & 10 & 1 & 13.5 \\
$72 \mathrm{~h}-160^{\circ} \mathrm{C}-\mathrm{PEG}_{10000}$ & 10 & 1 & 13.5 \\
\hline
\end{tabular}


It is suggested that the surface adsorption is a key point for the control of the growth process. The FTIR confirms that the binding of adsorbed surfactants on the surface of nanoparticles is weakened under the thermal conditions. The TGA analysis mainly showed a two-step loss at the two different ranges of temperature. By increasing the time and temperature of the process, the amount of loss was reduced.

\section{Appendix A. Supplementary material}

Supplementary data associated with this article can be found, in the online version, at http://dx.doi.org/10.1016/j.cej.2012.08.031.

\section{References}

[1] A.K. Gupta, A.S.G. Curtis, Surface modified superparamagnetic nanoparticles for drug delivery interaction studies with human fibroblasts in culture, J. Mater. Sci. - Mater. Med. 15 (2004) 493-496.

[2] R. Hergt, R. Hiergeist, I. Hilger, W.A. Kaiser, Y. Lapatnikov, S. Margel, U. Richter Maghemite nanoparticles with very high AC-losses for application in RFmagnetic hyperthermia, J. Magn. Magn. Mater. 270 (2004) 345-357.

[3] M. Johannsen, A. Jordan, R. Scholz, M. Koch, M. Lein, S. Deger, J. Roigas, K. Jung S. Loening, Evaluation of magnetic fluid hyperthermia in a standard rat model of prostate cancer, J. Endourol. 18 (2004) 495-500.

[4] R.H. Kodama, Magnetic nanoparticles, J. Magn. Magn. Mater. 200 (1999) 359.

[5] G.X. Li, V. Joshi, R.L. White, S.X. Wang, J.T. Kemp, C. Webb, R.W. Davis, S.H. Sun, Detection of single micron-sized magnetic bead and magnetic nanoparticles using spin valve sensors for biological applications, J. Appl. Phys. 93 (2003) 7557-7559.

[6] H. Zeng, J. Li, Z.L. Wang, J.P. Liu, S.H. Sun, Bimagnetic core/shell FePt/ $/ \mathrm{Fe}_{3} \mathrm{O}_{4}$ nanoparticles, Nano Lett. 4 (2004) 187-190.

[7] A. Chaudhuri, M. Mandal, K. Mandal, Preparation and study of $\mathrm{NiFe}_{2} \mathrm{O}_{4} / \mathrm{SiO}_{2}$ core-shell nanocomposites, J. Alloys Compd. 487 (2009) 698-702.

[8] J.F. Lee, Y.-M. Huh, Y.-M. Jun, J.-W. Seo, J.-T. Jang, H.-T. Song, S. Kim, E.-J. Cho, H.-G. Yoon, J.-S. Suh, Artificially engineered magnetic nanoparticles for ultrasensitive molecular imaging, J. Cheon. Nat. Med. 13 (2006) 95-99.

[9] B. Gillot, Fine-grained spinel ferrites: from the reactivity to magnetic properties, Eur. Phys. J. Appl. 4 (1998) 243-250.

[10] M. Sugimoto, The past, present and future of ferrites, J. Am. Ceram. Soc. 82 (1999) 269-280.

[11] Z.X. Tang, C.M. Sorensen, K.J. Klabunde, G.C. Hadjipanayis, Size-dependent curie temperature in nanoscale $\mathrm{MnFe}_{2} \mathrm{O}_{4}$ particles, Phys. Rev. Lett. 67 (1991) 3602-3605.

[12] G.U. Kulkarni, K.R. Kannan, T. Arunarkavalli, C.N.R. Rao, Particle-size effects on the value of $\mathrm{T}_{c}$ of $\mathrm{MnFe}_{2} \mathrm{O}_{4}$ : evidence for finite-size scaling, Phys. Rev. B 49 (1994) 724-727.

[13] B. Gillot, M. Laarj, S. Kacim, Reactivity towards oxygen and cation distribution of manganese iron spinel $\mathrm{Mn}_{3-x} \mathrm{Fe}_{x} \mathrm{O}_{4}(0 \leqslant x \leqslant 3)$ fine powders studied by thermogravimetry and IR spectroscopy, J. Mater. Chem. 7 (1997) 827-831.

[14] D. Zhang, X. Zhang, X. Ni, J. Song, H. Zheng, Low-temperature fabrication of $\mathrm{MnFe}_{2} \mathrm{O}_{4}$ octahedrons: magnetic and electrochemical properties, Chem. Phys. Lett. 426 (2006) 120-123.

[15] Y. Xia, Y. Xiong, B. Lim, S.E. Skrabalak, Shape-controlled synthesis of metal nanocrystals: simple chemistry meets complex physics?, Angew Chem. Int. Ed. 48 (2009) 60-103.

[16] S.G. Kwon, T. Hyeon, Colloidal chemical synthesis and formation kinetics of uniformly sized nanocrystals of metals, oxides, and chalcogenides, Acc. Chem. Res. (2008) 1696-1709.

[17] J. Park, J. Joo, S.G. Kwon, Y. Jang, T. Hyeon, Synthesis of monodisperse spherical nanocrystals, Angew. Chem. Int. Ed. 46 (2007) 4630-4660.

[18] N. Andersson, R.W. Corkery, P.C.A. Alberius, One-pot synthesis of well ordered mesoporous magnetic carriers, J. Mater. Chem. 17 (2007) 2700-2705.

[19] S. Yanez-Vilar, M. Sanchez-And ujar, C. Gomez-Aguirre, J. Mira, M.A. SenarısRodriguez, S.Castro-Garci, A simple solvothermal synthesis of $\mathrm{MFe}_{2} \mathrm{O}_{4}(\mathrm{M}=$ Mn, Co and Ni) nanoparticles, J. Solid State Chem. 182 (2009) 2685-2690.

[20] B. Aslibeiki, P. Kameli, H. Salamati, M. Eshraghi, T. Tahmasebi, Superspin glassstatein $\mathrm{MnFe}_{2} \mathrm{O}_{4}$ nanoparticles, J. Magn. Magn. Mater. 322 (2010) 29292934.

[21] J. Hu, I.M.C. Lo, G. Chen, Fast removal and recovery of $\mathrm{Cr}(\mathrm{VI})$ using surfacemodified Jacobsite $\left(\mathrm{MnFe}_{2} \mathrm{O}_{4}\right)$ nanoparticles, Langmuir 21 (2005) 1117311179.
[22] C. Liu, B. Zou, A.J. Rondinone, Z.J. Zhang, Reverse micelle synthesis and characterization of superparamagnetic $\mathrm{MnFe}_{2} \mathrm{O}_{4}$ spinel ferrite nanocrystallites, J. Phys. Chem. B 104 (2000) 1141-1145.

[23] C. Liu, Z.J. Zhang, Size-dependent superparamagnetic properties of Mn spinel ferrite nanoparticles synthesized from reverse micelles, Chem. Mater. 13 (2001) 2092-2096.

[24] S. Sun, H. Zeng, D.B. Robinson, S. Raoux, P.M. Rice, S.X. Wang, G. Li, Monodisperse $\mathrm{MFe}_{2} \mathrm{O}_{4}(\mathrm{M}=\mathrm{Fe}, \mathrm{Co}$., $\mathrm{Mn})$ nanoparticles, J. Am. Chem. Soc. 126 (2004) 273-279.

[25] S. Mohapatraa, S.R. Routa, A.B. Panda, One-pot synthesis of uniform and spherically assembled functionalized $\mathrm{MFe}_{2} \mathrm{O}_{4}(\mathrm{M}=\mathrm{Co}, \mathrm{Mn}, \mathrm{Ni})$ nanoparticles, Colloids Surf. A 384 (2011) 453-460.

[26] H. Zeng, P.M. Rice, S.X. Wang, S. Sun, Shape-controlled synthesis and shapeinduced texture of $\mathrm{MnFe}_{2} \mathrm{O}_{4}$ nanoparticles, J. Am. Chem. Soc. 126 (2004) $11458-11459$.

[27] W.-W. Wang, Microwave-induced polyol-process synthesis of $\mathrm{MIIFe}_{2} \mathrm{O}_{4}(\mathrm{M}=$, Co) nanoparticles and magnetic property, Mater. Chem. Phys. 108 (2008) 227231.

[28] J. Ge, Y. Hu, M. Biasini, W.P. Beyermann, Y. Yin, Superparamagnetic magnetite colloidal nanocrystal clusters, Angew. Chem. Int. Ed. 46 (2007) 4342-4345.

[29] H. Deng, X. Li, Q. Peng, X. Wang, J. Chen, Y. Li, Monodisperse magnetic singlecrystal ferrite microspheres, Angew. Chem. Int. Ed. 44 (2005) 2782-2785.

[30] J. Liu, Z. Sun, Y. Deng, Y. Zou, C. Li, X. Guo, L. Xiong, Y. Gao, F. Li, D. Zhao, Highly water-dispersible biocompatible magnetite particles with low cytotoxicity stabilized by citrate groups, Angew. Chem. Int. Ed. 48 (2009) 5875-5879.

[31] J. Zhang, Y. Wang, J. Zheng, F. Huang, D. Chen, Y. Lan, G. Ren, Z. Lin, C. Wang Oriented attachment kinetic for ligand capped nanocrystals: coarsening of thiol-PbS nanoparticles, J. Phys. Chem. B 111 (2007) 1449-1454.

[32] Y. Wang, J. Zhang, Y. Yang, F. Huang, J. Zheng, D. Chen, F. Yan, Z. Lin, C. Wang, $\mathrm{NaOH}$ concentration effect on the oriented attachment growth kinetics of $\mathrm{ZnS}$ J. Phys. Chem. B 111 (2007) 5290-5294.

[33] J.F. Banfield, A. Navrotsky, Nanoparticles in Environment, Mineralogical Society of America, 2001. p. 44

[34] R.L. Penn, Kinetics of oriented aggregation, J. Phys. Chem. B 108 (2004) 1270712712 .

[35] R.L. Penn, K. Tanaka, J. Erbs, Size dependent kinetics of oriented aggregation, J. Cryst. Growth 309 (2007) 97-102.

[36] M. Niederberger, H. Coelfen, Oriented attachment and mesocrystals: nonclassical crystallization mechanisms based on nanoparticle assembly, Phys. Chem. Chem. Phys. 8 (2006) 3271-3287.

[37] Q.Song. R-, H. Colfen, Mesocrystals-ordered nanoparticle superstructures, Adv Mater. 22 (2010) 1301-1330.

[38] O. Pujol, P. Bowen, P.A. Stadelmann, H. Hofmann, Growth and self-assembly of nanostructured $\mathrm{CoC}_{2} \mathrm{O}_{4} \cdot 2 \mathrm{H}_{2} \mathrm{O}$ particles, J. Phys. Chem. B 108 (2004) 1312813136.

[39] Z. Zhang, H. Sun, X. Shao, D. Li, H. Yu, M. Han, Three-dimensionally oriented aggregation of a few hundred nanoparticles into monocrystalline architectures, Adv. Matter. 17 (2005) 42-47.

[40] H. Colfen, M. Antonietti, Mesocrystals: inorganic superstructures made by highly parallel crystallization and controlled alignment, Angew. Chem. Int. Ed. 44 (2005) 5576-5591.

[41] B.D. Cullity, Elements of X-ray Diffraction, second ed., Addison Wesley, 1978.

[42] B. Luo, X.-J. Song, F. Zhang, A. Xia, W.-L. Yang, J.-H. Hu, C.-C. Wang, Multifunctional thermosensitive composite microspheres with high magnetic susceptibility based on magnetite colloidal nanoparticle clusters, Langmuir 26 (2010) 1674-1679.

[43] H.G. Yang, H.C. Zeng, Preparation of hollow anatase $\mathrm{TiO}_{2}$ nanospheres via Ostwald ripening, J. Phys. Chem. B 108 (2004) 3492-3495.

[44] R.D. Palma, S. Peeters, M.J. Van Bael, H. Van den Rul, K. Bonroy, W. Laureyn, J. Mullens, G. Borghs, G. Maes, Silane ligand exchange to make hydrophobic superparamagnetic nanoparticles water-dispersible, Chem. Mater. 19 (2007) 1821-1831.

[45] Y. Sahoo, H. Pizem, T. Fried, D. Golodnitsky, L. Burstein, C.N. Sukenik, G. Markovich, Alkyl phosphonate/phosphate coating on magnetite nanoparticles: a comparison with fatty acids, Langmuir 17 (2001) 7907-7911.

[46] A. Swami, A. Kumar, M. Sastry, Formation of water-dispersible gold nanoparticles using a technique based on surface-bound interdigitated bilayers, Langmuir 19 (2003) 1168-1172.

[47] C. Yee, G. Kataby, A. Ulman, T. Prozorov, H. White, A. King, M. Rafailovich, J. Sokolov, A. Gedanken, Self-assembled monolayers of alkanesulfonic and phosphonic acids on amorphous iron oxide nanoparticles, Langmuir 15 (1999) 7111-7115

[48] L. Zhang, R. He, H.-C. Gu, Oleic acid coating on the monodisperse magnetite nanoparticles, App. Surf. Sci. 253 (2006) 2611-2617. 